\title{
Mesopic radial frequency contrast sensitivity function for young and older adults
}

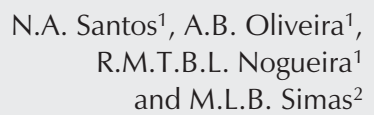

\author{
${ }^{1}$ Departamento de Psicologia, Universidade Federal da Paraíba, João Pessoa, \\ PB, Brasil \\ ${ }^{2}$ Departamento de Psicologia, Universidade Federal de Pernambuco, Recife, \\ PE, Brasil
}

\author{
Correspondence \\ N.A. Santos \\ Departamento de Psicologia \\ CCHLA, UFPB \\ 58051-900 João Pessoa, PB \\ Brasil \\ Fax: +55-83-3216-7064 \\ E-mail: natanael_labv@yahoo.com.br \\ Presented at the XX Annual Meeting \\ of the Federação de Sociedades de \\ Biologia Experimental, \\ Águas de Lindóia, SP, Brazil, \\ August 24-27, 2005. \\ Research supported by CNPq (No. \\ 40.3211/2003-4). N.A. Santos and \\ M.L.B. Simas are recipients of \\ CNPq productivity fellowships \\ (Nos. 307182/2003-7 and \\ 35.1453/1996-2, respectively).
}

\begin{abstract}
The objective of the present study was to determine contrast sensitivity curves of concentric circular patterns with radial frequencies of $0.25,0.5,1.0,2.0$, and 4.0 cycles per degree in young and older adult volunteers. These parameters were also compared with sensitivity contrasts for sine-wave gratings. All participants had normal acuity vision and were free of identifiable ocular illness. Contrast sensitivity was measured in 6 young adults aged 19 to 23 years and 6 older adults aged 60 to 69 years using the psychophysical forced-choice method. In this paradigm the volunteers had to decide which of two stimuli contained the above radial frequencies at low contrast levels. The other neutral stimulus was gray with homogeneous luminance. We detected a decline in contrast sensitivity for older adults at all radial frequencies compared to young adults. Also, contrast sensitivity for sine-wave gratings at all measured frequencies was better, as predicted, for all young adults. Maximum sensitivities in the radial frequency contrast sensitivity function and contrast sensitivity function occurred at 0.25 and 0.5 cycles per degree, respectively, for both young and older adults. These results suggest age-related changes in the contrast sensitivity function for concentric symmetrical stimuli.
\end{abstract}

Key words

- Visual form processing

- Radial frequency

- Contrast sensitivity

- Forced-choice method
Alterations in the optic and neural characteristics of the human visual system occurring with age have been the subject of several studies which have tried to relate these alterations to the contrast sensitivity functions (CSF) for vertical sine-wave gratings and angular frequency stimuli defined in Cartesian and polar coordinates, respectively (1-7). Although there is no general consensus about the frequency ranges that are affected by aging, psychophysical studies on humans using photopic conditions found i) reduction of contrast sensitivity in medium and high spatial and angular frequencies with age, and ii) significant declines in CSF, only when volunteers were 50 years old (3,6-10).

In the present study, we determined the CSF for concentric circular patterns, i.e., radial frequency CSF stimuli (rCSF) for young and old adults, under mesopic conditions using a forced-choice psychophysical method. Radial frequency stimuli are defined in polar coordinates, where the spatial frequency varies along the radius and is 
modulated by spherical Bessel functions, $\mathrm{j}_{0}$ (see details in Refs. 11,12). They are referred to in the literature as $\mathrm{J}_{0}$ targets (cylindrical Bessel functions), Bull's eye or circular gratings (11). Contrary to sine-wave gratings, radial frequency stimuli are circularly symmetric and have a fixed center.

The aim of the present study was to relate the human aging process to the rCSF, particularly at low luminance levels. Studies on human vision measuring sensitivity to radial frequency stimuli are dispersed, have rarely been conducted on young adults (11-13) and are non-existent for the older population. Six young adults (19 to 23 years old) and six older adults (60-69 years old) participated in the present investigation. They were free of ocular diseases and had been examined by an ophthalmologist during the last 12 months. Both groups had normal or corrected to normal acuity vision.

Stimuli were generated on a CRT-based Clinton Medical digital monochromatic video monitor (New York, NY, USA) with a 21inch display, high resolution (1280 x 1024), a $75-\mathrm{Hz}$ frame rate, and with input controlled by a microcomputer. Measurements were made at a distance of $150 \mathrm{~cm}$ from the monitor screen. Mean luminance was $0.5 \mathrm{~cd} /$ $\mathrm{m}^{2}$ measured with an Asahi Pentax $1^{\circ}$ spot meter (New York, NY, USA). The minimum and maximum luminance values were 0.3

Figure 1. Examples of radial frequency stimuli (e.g., 0.25, 0.5, 1.0 , and $2.0 \mathrm{cpd}$ ) which were originally calibrated to be seen at a distance of $150 \mathrm{~cm}$ on the 21 " display.

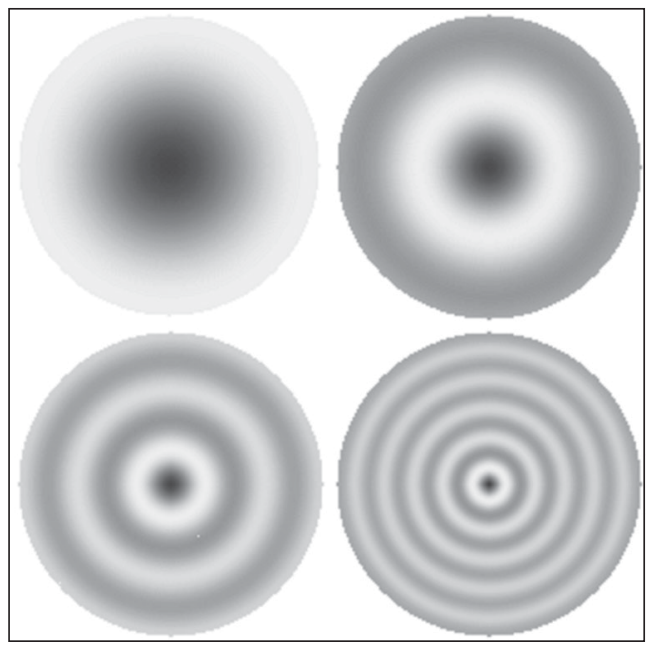

and $0.7 \mathrm{~cd} / \mathrm{m}^{2}$. The walls of the room were gray for better control of ambient luminance.

The achromatic radial frequency stimuli used to measure the rCSF were $0.25,0.5$, $1.0,2.0$, or 4.0 cycles per degree (cpd) of visual angle. The aperture was circular and had a diameter of 7 degrees of visual angle (Figure 1).

Measurements were made using a temporal two-alternative forced-choice method (similar to those found in Refs. 5,6,11, and 13). This method is based on the probability of consecutive correct choices made by participants following the presentation of two stimuli, in this case, one at mean luminance $\left(0.5 \mathrm{~cd} / \mathrm{m}^{2}\right.$, neutral stimulus) and the other containing one of the radial frequencies. The correct choice was to select the radial frequency stimuli (first or second of each pair). The number of consecutive correct answers required to lower contrast by one unit $(0.08 \%)$ was set to three. Contrast was always increased by the same unit after each incorrect choice. This yielded a probability of perceiving the radial frequency stimuli set at $79 \%$.

An experimental session started with a brief beep followed by the presentation of a given test stimulus for $2 \mathrm{~s}$, followed by an inter-stimulus interval of $1 \mathrm{~s}$ and by the presentation of the other stimulus for $2 \mathrm{~s}$, followed by the volunteer's response. The inter-trial interval was $3 \mathrm{~s}$. A different beep gave feedback about each correct answer. The duration of an experimental session depends on the responses given by the participant and ended only after 5 pairs of peaks and valleys had been obtained (i.e., 10 point estimate values). Duration usually was about 10-15 min.

Each point estimate was measured at least twice, always on different days, for each participant. A total of twelve functions (of five point estimates each) was measured for both groups of participants, yielding a sample of about 120 point estimates for each radial frequency stimulus under measurement. 
Figure 2 shows the rCSF for young and older adults. Contrast sensitivity estimates are shown as a function of radial frequencies. The standard error of the mean (error bars) was corrected for sample size to represent the $99 \%$ confidence interval.

The functions (rCSF) for both groups showed maximum sensitivity at the radial frequency of $0.25 \mathrm{cpd}$, i.e., the lowest frequency, but the older age group needed 2.2 times more contrast than the young adult group (Figure 2A). Older adults needed 2.1, $1.5,1.4$, and 1.4 times more contrast than young adults at the remaining radial frequencies, i.e., at $0.5,1.0,2.0$, and $4.0 \mathrm{cpd}$, respectively (Figure $2 \mathrm{~A}$ ).

One-way ANOVA showed a significant difference between young and older adults $\left(\mathrm{F}_{9,1119}=22.15 ; \mathrm{P}<0.001\right)$. The post hoc Tukey HSD test applied to the rCSF data showed that the differences between groups were statistically significant at all radial frequencies (i.e., $\mathrm{P}<0.001$ for $0.25 \mathrm{cpd}, \mathrm{P}<$ 0.001 for $0.5 \mathrm{cpd}, \mathrm{P}<0.001$ for $1.0 \mathrm{cpd}, \mathrm{P}<$ 0.001 for $2.0 \mathrm{cpd}$, and $\mathrm{P}<0.003$ for $4 \mathrm{cpd}$ ).

These data show that the mesopic sensitivity to radial frequencies was higher for young adults at all frequencies compared to older adults.

The main purpose of this research was to characterize the visual systems of young and older adults by measuring the rCSF under low luminance levels and to evaluate possible visual process changes with aging. The data showed that this function is different for young (19-23 years old) and older adults (60-69 years old). There was a decrease in sensitivity with age at all radial frequencies, similar to that found for spatial frequencies in the literature $(4,8-10,14-16)$. The use of lower level contrast (i.e., medium luminance of $0.5 \mathrm{~cd} / \mathrm{m}^{2}$ ) explains in some way the displacement of the maximum sensitivity range towards the very low radial frequency (i.e., $0.25 \mathrm{cpd}$ ). The CSF shape changes according to viewing conditions $(8,9)$.

We also measured the mesopic CSF for spatial frequencies using sine-wave gratings (i.e., $0.25,0.5,1.0,2.0$, and $4.0 \mathrm{cpd}$ ) with the same participants, the same method and equivalent experimental conditions (Figure 2B). Our results for the CSF under these conditions are similar to those reported in the literature that show less contrast sensitivity for older adults at all spatial frequencies $(8-10,16)$. These results also show a similar trend in the data for radial frequencies (Figure 2A). One-way ANOVA showed a significant difference between young and older adults $\left(\mathrm{F}_{9,1119}=125.9 ; \mathrm{P}<0.001\right)$. Analyses applying the post hoc Tukey HSD test to contrast sensitivity function data showed that all differences between groups were statistically significant (i.e., $\mathrm{P}<0.046$ for $0.25 \mathrm{cpd}, \mathrm{P}<0.001$ for $0.5 \mathrm{cpd}, \mathrm{P}<0.001$ for $1.0 \mathrm{cpd}, \mathrm{P}<0.001$ for $2.0 \mathrm{cpd}$, and $\mathrm{P}<$ 0.001 for $4.0 \mathrm{cpd}$ ).

Therefore, it is possible that aging alters the nervous system in a diffuse way (17), with changes in the mechanisms involved in the processing of spatial frequency and radial frequency stimuli. The available literature suggests that these stimuli (i.e., radial frequency and sine-wave gratings) are most

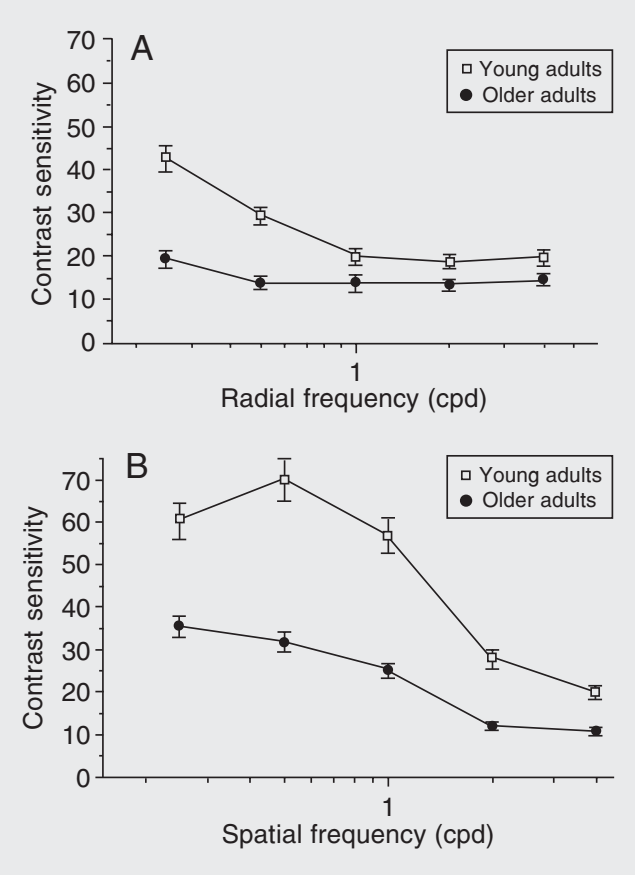

Figure 2. Radial frequency sensitivity functions for young and older adults (A) and spatial frequency contrast sensitivity (for vertical sine-wave gratings) for the two groups (B). Data are means \pm SEM or SD for 6 subjects in each group. Error bars are corrected to represent the $99 \%$ confidence interval. $\mathrm{cpd}=$ cycles per degree. 
likely processed by different visual cortical areas (for instances, see Refs. 11,13,18-20). Our results are preliminary and we are still running additional related experiments to better establish the effects of aging on the visual processing of radial frequencies.

All measurements were approved by the University Ethics Committee and written informed consent was obtained from each volunteer.

\section{References}

1. Arden GB, Jacobson JJ. A simple grating test for contrast sensitivity: preliminary results indicate value in screening for glaucoma. Invest Ophthalmol Vis Sci 1978; 17: 23-32.

2. Arundale K. An investigation into the variation of human contrast sensitivity with age and ocular pathology. Br J Ophthalmol 1978; 62: 213-215.

3. Elliott D, Whitaker D, MacVeigh D. Neural contribution to spatiotemporal contrast sensitivity decline in healthy ageing eyes. Vision Res 1990; 30: 541-547.

4. McGrath C, Morrison JD. The effects of age on spatial frequency perception in human subjects. $Q J$ Exp Physiol 1981; 66: 253-261.

5. Owsley C, Sekuler R, Siemsen D. Contrast sensitivity throughout adulthood. Vision Res 1983; 23: 689-699.

6. Sekuler R, Hutman LP, Owsley CJ. Human aging and spatial vision. Science 1980; 209: 1255-1256.

7. Fiorentini A, Porciatti V, Morrone MC, Burr DC. Visual ageing: unspecific decline of the responses to luminance and colour. Vision Res 1996; 36: 3557-3566.

8. Schefrin BE, Tregear SJ, Harvey Jr LO, Werner JS. Senescent changes in scotopic contrast sensitivity. Vision Res 1999; 39: 37283736.

9. Higgins KE, Jaffe MJ, Caruso RC, deMonasterio FM. Spatial contrast sensitivity: effects of age, test-retest, and psychophysical method. J Opt Soc Am A 1988; 5: 2173-2180.

10. Nio YK, Jansonius NM, Fidler V, Geraghty E, Norrby S, Kooijman AC. Age-related changes of defocus-specific contrast sensitivity in healthy subjects. Ophthalmic Physiol Opt 2000; 20: 323-334.

11. Simas ML, Santos NA, Thiers FA. Contrast sensitivity to angular frequency stimuli is higher than that for sinewave gratings in the respective middle range. Braz J Med Biol Res 1997; 30: 633-636.

12. Simas ML, Santos NA. Narrow-band 1, 2, 3, 4, 8, 16, and 24 cycles/ 360 degrees angular frequency filters. Braz J Med Biol Res 2002; 35: 243-253.

13. Crassini B, Brown B, Bowman K. Age-related changes in contrast sensitivity in central and peripheral retina. Perception 1988; 17: 315332.

14. Elliott DB. Contrast sensitivity decline with ageing: a neural or optical phenomenon? Ophthalmic Physiol Opt 1987; 7: 415-419.

15. Ross JE, Clarke DD, Bron AJ. Effect of age on contrast sensitivity function: uniocular and binocular findings. Br J Ophthalmol 1985; 69: 51-56.

16. Jackson GR, Owsley C, Cordle EP, Finley CD. Aging and scotopic sensitivity. Vision Res 1998; 38: 3655-3662.

17. Habak C, Faubert J. Larger effect of aging on the perception of higher-order stimuli. Vision Res 2000; 40: 943-950.

18. Gallant JL, Connor CE, Rakshit S, Lewis JW, Van Essen DC. Neura responses to polar, hyperbolic, and Cartesian gratings in area V4 of the macaque monkey. J Neurophysiol 1996; 76: 2718-2739.

19. Merigan WH. Basic visual capacities and shape discrimination after lesions of extrastriate area V4 in macaques. Vis Neurosci 1996; 13: 51-60.

20. Wilkinson F, James TW, Wilson HR, Gati JS, Menon RS, Goodale MA. An fMRI study of the selective activation of human extrastriate form vision areas by radial and concentric gratings. Curr Biol 2000; 10: 1455-1458. 